\title{
Protocol
}

\section{Routes of Antigen Injection in Rabbits}

\author{
Edward A. Greenfield
}

MATERIALS

The route of antigen injection is guided by three practical decisions: (1) the volume to be delivered, (2) the buffers and other components to be injected with the immunogen, and (3) how quickly the immunogen should be released into the lymphatics or circulation. For rabbits, large-volume injections normally are given at multiple subcutaneous sites. If adjuvants or particulate matter are included in the injection, the immunogen should not be delivered intravenously. If slow release of the inoculum is desired, the injections should be performed either intramuscularly or intradermally. For immediate release, use intravenous injections.

It is essential that you consult the appropriate Material Safety Data Sheets and your institution's Environmental Health and Safety Office for proper handling of equipment and hazardous materials used in this protocol.

Adjuvant (optional)

Anesthesia (optional)

Antigen of interest

Rabbits to be immunized

Equipment

Cotton or gauze, sterile

Needles (25 gauge)

Restraining device (for intravenous injections; see Step 21)

Shaver (for intradermal injections; see Step 14)

Syringes

For most injections, disposable syringes are best. Plastic syringes are appropriate for all injections except when using Freund's adjuvant, in which case, disposable glass syringes are recommended. Syringes can be purchased with several different tips. The most useful are the Luer tip and Luer lock. For most work, the Luer tip is fine, but use the Luer lock for samples that are viscous. After removing the syringe from its container, push the plunger in completely to loosen the seal that has formed between the plunger and the barrel.

Before beginning an immunization, contact your local safety and animal committees for advice on animal care and handling, local regulations, and proper procedures for immunization.

From the Antibodies collection, edited by Edward A. Greenfield.

(C) 2018 Cold Spring Harbor Laboratory Press

Cite this protocol as Cold Spring Harb Protoc; doi:10.1101/pdb.prot100222 


\section{Injecting Rabbits Subcutaneously}

Subcutaneous injections are easily performed in rabbits. Their skin is loose, allowing for larger volumes of material to be injected. They are normally given multiple subcutaneous injections on the back. Injections can include both particulate immunogens and/or adjuvants. Injected material will drain quickly into the local lymphatic system and will become concentrated in the lymph nodes closest to the injected sites. Injections should not be performed at the neck because rabbits are usually picked up by the neck during handling. Anesthesia is normally recommended to allow easier handling during injections.

1. Anesthetize the rabbit (see Protocol: Administering Anesthesia to Rabbits [Greenfield 2018]) or check with staff veterinarian for protocol).

2. Starting on one side near the back of the neck, pinch the skin between the thumb and forefinger. Pull the skin away from the body. Insert the 25-gauge needle into the space that has been created (Fig. 1).

3. Move the end of the needle a short distance from side-to-side to ensure that it is not inserted into muscle or the body wall.

4. Depress the plunger to inject the desired amount of solution. (Use $\sim 400 \mu \mathrm{L}$ of immunogen or immunogen-adjuvant solution for a normal subcutaneous injection). Pause a few seconds.

5. Withdraw the needle, gently rubbing the hole with the forefinger and thumb to stop any of the inoculum from escaping.

6. Move to the next site and repeat.

Up to 10 sites per animal are common.

\section{Injecting Rabbits Intramuscularly}

Intramuscular injection is a popular strategy for immunizing rabbits. It is one of the routes of inoculation used to produce a slow release of an antigen. Both adjuvant and particulate inoculi can be used. The inoculum is deposited directly into the muscle tissue. Smaller-molecular-weight antigens will enter the bloodstream for distribution, and larger-molecular-weight antigens will be absorbed into the lymphatic system.

7. Draw the antigen into a syringe. Use a 25 -gauge needle for the injection.

A normal intramuscular injection is $200-400 \mu \mathrm{L}$.

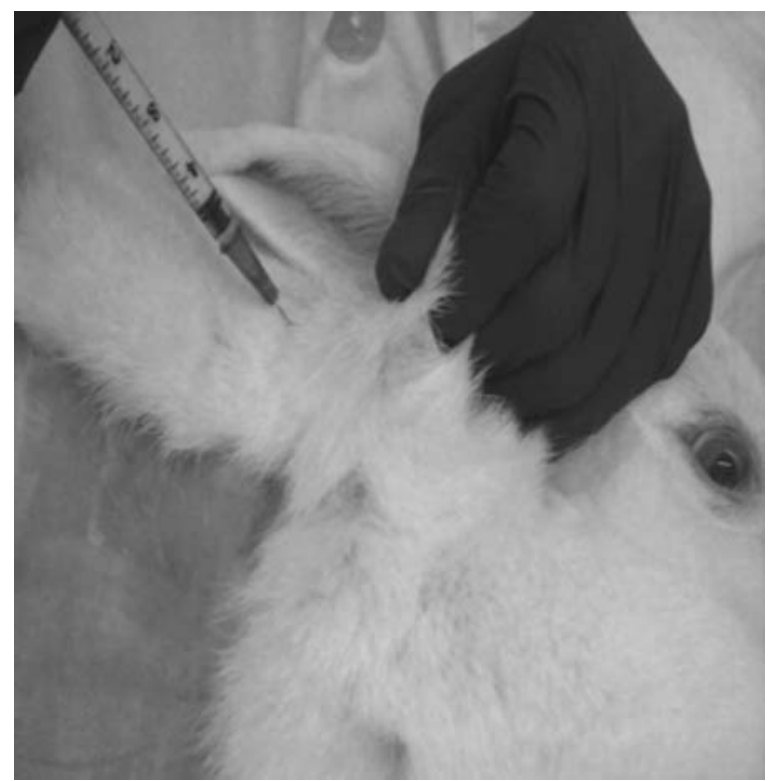

FIGURE 1. Subcutaneous injection of a rabbit. (Photo courtesy of Millbrook Immunoserv, Amherst, MA.) 
E.A. Greenfield

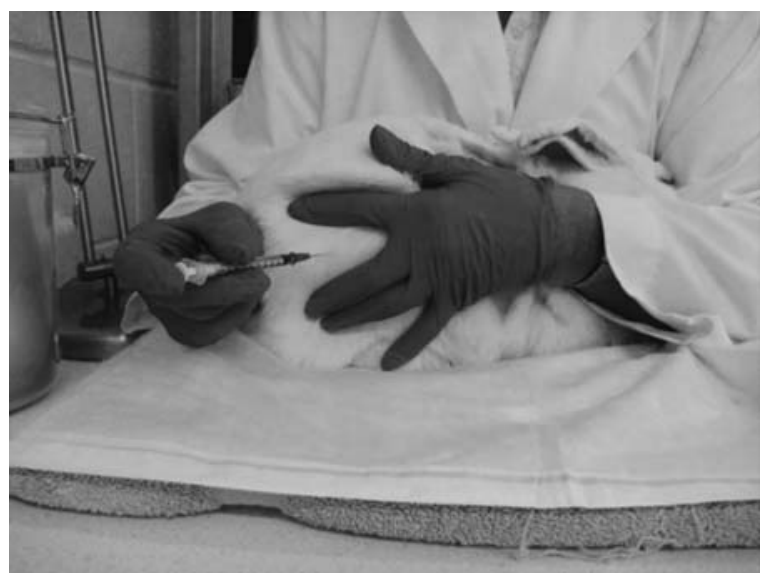

FIGURE 2. Intramuscular injection into a rabbit. (Photo courtesy of Millbrook ImmunoServ, Amherst, MA.)

8. Remove the rabbit from its cage. Place it on a wire or solid surface that is rough enough for the rabbit to feel comfortable.

Avoid the laboratory bench because the rabbit will constrict its muscles on any smooth surface. Wrapping the rabbit in a towel is often sufficient, and this can serve as a restricting device.

9. If working alone, anesthetize the rabbit before injection (see Protocol: Administering Anesthesia to Rabbits [Greenfield 2018]). If assistance is available to immobilize the rabbit's leg, proceed with the injection without anesthesia.

10. Grasp the rabbit from the front so that one of the rear legs is accessible. Make the injection into the rear of the thigh muscle near the hip (Fig. 2).

11. Insert the needle into the muscle, and gently try to withdraw the plunger. If there is resistance, proceed with the injection. If blood appears, the needle has found one of the small veins or arteries of the leg. Withdraw the syringe, move to a nearby site, and try again.

12. Inject the inoculum slowly and in a steady motion. Withdraw the needle. Gently massage the site of injection.

Injecting Rabbits Intradermally

Intradermal (i.d.) injections offer the advantage of a slow absorption rate into the bloodstream but a rapid uptake into the lymphatic system (Vaitukaitis et al. 1971; Vaitukaitis 1981). The inoculum is injected between the layers of the skin. Langerhans cells in the dermis are present to take up the antigen and present it to immune cells in the draining lymph nodes. Intradermal injections can be used with particulate- and adjuvant-containing inoculi. Under no circumstances should Complete Freund's adjuvant be used for more than the first injection when using intradermal injections. Only small injection volumes can be used; thus, intradermal injection is only suitable if the antigen is available in a concentrated form. It is normal practice to deliver the injection over multiple sites. The procedure requires more skill than other injections.

13. Anesthetize a rabbit before injection.

14. Shave an appropriately sized area to expose the skin either high on the flank (between the ribs and hip) or across the back (Fig. 3).

15. Hold the skin between the forefinger and thumb.

16. Check to be certain that the 25-gauge needle is secured tightly to the syringe. Insert the needle with the bevel side up slightly under the skin. Continue pushing the needle between the layers of skin, and guide it forward for at least $0.5-0.7 \mathrm{~cm}$.

17. Inject no more than $100 \mu \mathrm{L}$.

The inoculum should form a tight blister under the skin. 

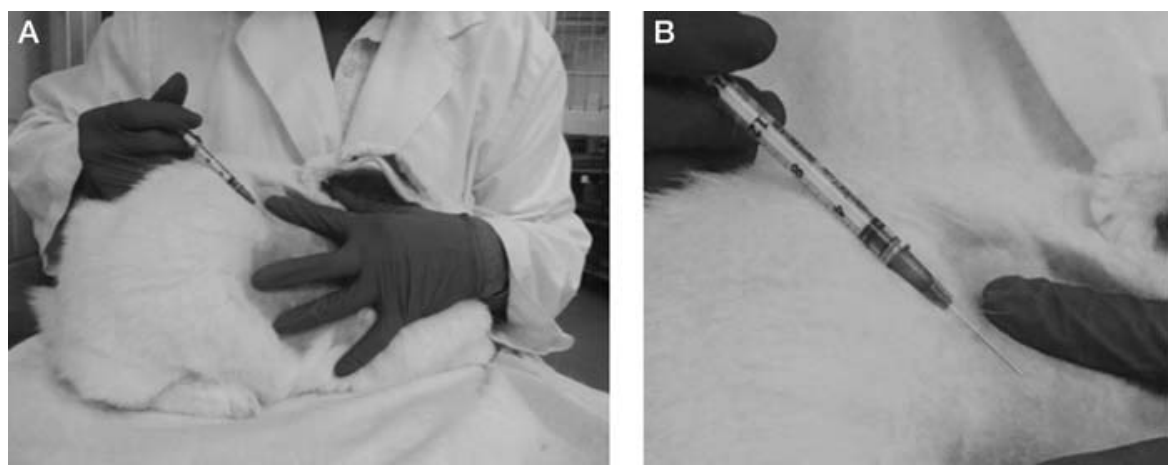

FIGURE 3. Intradermal injection into a rabbit. (A) Injection. (B) Close-up. (Photos courtesy of Millbrook ImmunoServ, Amherst, MA.)

18. Withdraw the needle, holding the needle track with the thumb and forefinger. Compress the skin gently as the needle comes out.

19. Repeat the procedure at the next site. Injecting at up to 40 sites is common.

If Complete Freund's adjuvant is included, small sores will appear at the sites of injection. These will clear within a few weeks.

\section{Injecting Rabbits Intravenously}

The best route of injection for soluble antigens without adjuvant is intravenous (i.v.). It delivers the antigen efficiently to lymphoid organs like the spleen and lymph nodes. However, there is a risk of anaphylactic shock (although this can be prevented by a prior injection of an antihistamine). Also, without adjuvant there is no depot effect. Thus, intravenous injection is not usually used as the primary route of immunization. In rabbits, intravenous injections are normally performed in the marginal ear vein.

20. Draw the antigen into a syringe. Use a 25-gauge needle for the injection.

A normal intravenous injection is 500-1000 $\mu \mathrm{L}$.

21. Remove the rabbit from its cage. Place it in a restraining device.

22. Pull the ear out straight. The vein is on the far inside edge of the ear. Press lightly on the base of the ear to restrict return of the blood to the body; this will make the vein stand out strongly.

23. Insert the needle into the vein (Fig. 4). Gently pull back on the plunger. If blood appears in the syringe, proceed with the injection. If no blood is seen, move to another location on the vein and try again.
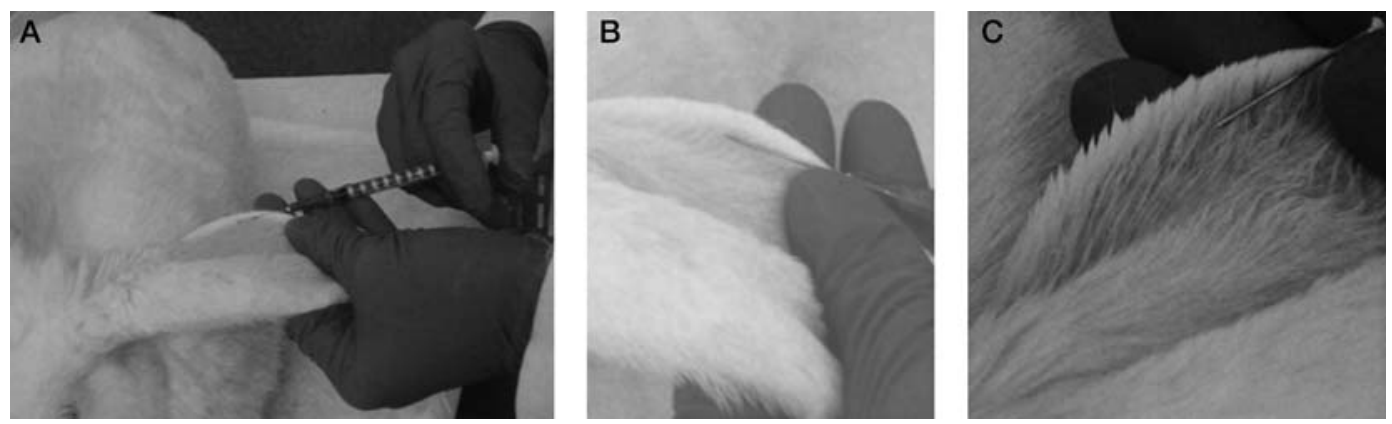

FIGURE 4. Intravenous injection into a rabbit. (A) Injection. (B) Close-up. (C) Intra-arterial injection (for euthanasia or antigen immunization without adjuvant). (Photos courtesy of Millbrook ImmunoServ, Amherst, MA.) 
E.A. Greenfield

24. Slowly and evenly inject the antigen solution into the vein. Pause for a few seconds.

25. Remove the needle. Place a piece of cotton or sterile gauze over the injection site as the needle is removed. Hold the site tightly for a few seconds to stop any bleeding.

\section{REFERENCES}

Greenfield EA. 2018. Administering anesthesia to rabbits. Cold Spring Harb Protoc doi: $10.1101 /$ pdb.prot 100206.

Vaitukaitis JL. 1981. Production of antisera with small doses of immunogen: Multiple intradermal injections. Methods Enzymol 73: 46-52.
Vaitukaitis J, Robbins JB, Nieschlag E, Ross GT. 1971. A method for producing specific antisera with small doses of immunogen. J Clin Endocrinol Metab 33: 988-991. 


\section{Routes of Antigen Injection in Rabbits}

Edward A. Greenfield

Cold Spring Harb Protoc; doi: 10.1101/pdb.prot100222

\begin{tabular}{rc}
$\begin{array}{r}\text { Email Alerting } \\
\text { Service }\end{array}$ & Receive free email alerts when new articles cite this article - click here. \\
\hline $\begin{array}{r}\text { Subject } \\
\text { Categories }\end{array}$ & $\begin{array}{c}\text { Browse articles on similar topics from Cold Spring Harbor Protocols. } \\
\text { Antibodies (119 articles) } \\
\text { Generating Antibodies (71 articles) } \\
\text { Generating Antibodies, general (60 articles) }\end{array}$ \\
\hline
\end{tabular}

